\title{
4 Communication in Families with Adolescents
}

The quality of the communication between family members is critical to the quality of family relationships and to adolescent adjustment. In this chapter we aim to reflect the findings of the large amount of research that has been carried out in the field of family communication over the last few decades. We aim to explore the issues for adolescents as they seek to maintain positive relationships with their parents while they mature physically, cognitively and socially as individuals. During this stage they also strive to develop their individual identities and fulfil their needs for more autonomy, while maintaining their position within the family. The development of the communication between parents and their adolescent is very important to this process of individuation (e.g. Keijsers \& Poulin, 2013).

Whatever the life stage of the family, the importance of communication cannot be overestimated and communication may be particularly crucial for families with adolescents. Communication between adolescents and their parents can, at times, be difficult, but open and constructive communication is critical to a healthy family environment and to the well-being of the adolescents (Noller, 2005a.).

\subsection{The changing nature of parent-adolescent relationships}

As discussed in Chapter 2, one of the main developmental tasks for adolescents is to become independent from parents (Robin \& Foster, 1989). During this stage adolescents need to develop their own opinions, become aware of those of others, and be able to integrate the two (Grotevant\& Cooper, 1986; Keijsers \& Poulin, 2013; see Chapter 2 for further discussion of adolescent individuation and autonomy). Individuation occurs within a relational context and involves a combination of the adolescent striving for individuality while at the same time wanting to maintain connectedness to relationships in the family. Successful individuation by adolescents involves their being able to express their own points of view, including opinions that may differ from those of their parents, while also being sensitive to and respectful of the opinions of others (Grotevant\& Cooper, 1985). This situation creates challenges to the existing family structure, whatever that structure (Robin \& Foster, 1989), with the parent-child relationship undergoing transformation from one that is relatively ordered with the parent in the dominant role, to one that involves a more equal relationship between parents and adolescents (Flannery, Montemayor, Eberly \& Torquati, 1993; Grotevant \& Cooper, 1986; Hunter, 1985; Hunter \& Youniss, 1982; Rosnati, Iafrate \& Scabini, 2007; Steinberg, 1990).

Just as adolescents need to change the ways they communicate with their parents, parents also need to change their communication with their adolescent in terms of how much independence they allow to their adolescent, or expect of them (Goossens, 2006a). How often parents and adolescents talk with one another is likely to change 
over time, as are the topics of their conversations with changing expectations of privacy and responsibility, and the changing nature of the experiences they share (Laursen \& Collins, 2004).

Because of the importance of communication, researchers have investigated how parents and adolescents deal with issues that create conflict within the family. One reason for a strong focus on conflict is that adolescence is a stage when offspring are 'pushing the boundaries' in a search for greater autonomy over their lives (see Chapter 2). In addition, adolescents are still establishing their values and are eager to try new experiences and work out their own beliefs and values. In this chapter, we also explore the role of conflict in adolescent development and the importance of parental response and guidance in these situations.

A critical issue for adolescents concerns who should control the various aspects of their lives, and conflict often arises because adolescents come to believe that areas that were formerly under the control of parents should now be under their own control (Steinberg \& Silk, 2002). For this reason, they expect to be included in decisions that affect them, and to be accepted and encouraged rather than criticised by their parents.

Modern communication technologies have also had an impact on communication in families. A lot of communication with peers takes place using mobile phones or through the internet. Time spent in these activities and time spent 'surfing' the internet can all take time away from communication with the family. In addition, adolescents can engage in relationships, even risky relationships, which parents have difficulty monitoring. Parents may also have difficulty monitoring adolescents' exposure to sex and violence on the web and on TV.

\subsection{Parent-Adolescent Communication}

The ways that parents and adolescents interact and communicate with each other is important for both their relationships and for adolescent well being (e.g., Collins \& Laursen, 2004) and for family functioning in general (Lila, van Aken, Musitu \& Buelga, 2006). Adolescents have a unique relationship with parents and when these relationships are positive, a strong bond develops based on years of knowledge about each other that contributes to a shared understanding of each others' communication (Lewis, Wallerstein, \& Johnson-Reitz, 2004; Sillars, Koerner \& Fitzpatrick, 2005).

Not only is communication important to family relationships, positive parentadolescent communication facilitates the development of adolescents' social and leadership skills and enhances their resilience in problematic situations (LeBlanc, Self-Brown \& Shepard, 2011). Having dinner together as often as possible seems to facilitate communication among family members and contributes to adolescents' positive perceptions of their communication with parents (Fulkerson, Pasch, Stigler, Farbakhsh, Perry \& Komro, 2010; Offer, 2013; Skeer \& Ballard, 2013). 
In a review of the literature regarding US studies, Skeer and Ballard (2013) examined the findings about the associations between the frequency of shared family mealtimes and adolescents' risky behaviour. The literature review indicated that more frequent family mealtimes are associated with better school performance, healthier eating patterns, delayed sexual activity and better mental health outcomes. The findings concerning substance use were mixed across studies and varied depending on gender and type of substance. Although these authors highlight the limitations of the literature their findings do suggest that shared mealtimes are a protective factor for adolescents across a range of issues. Limitations mentioned by Skeer and Ballard include: the definition of meal-time in the studies and the lack of information about the processes, or how the frequency of family mealtimes may be beneficial for positive adolescent development.

Fulkerson et al.'s longitudinal US study included African-American, Latino and white adolescents and involved young people's reports of their communication with their parents over a three and a half year period. African-Americans reported significantly fewer family dinners at baseline than other adolescents and across racial groups. Adolescents in 2-parent families reported more frequent family dinners than those in other types of families. The study showed that sharing dinner-time increases the likelihood of more family communication that includes parental praise of the adolescent, monitoring of their activities and discussion about their education. It is important to keep in mind that this sample was dominated by African-American families and the findings may not apply as clearly to other kinds of families.

Offer (2013) looked specifically at family communication during mealtimes. The results of this study indicated that family communication was greater when both parents were present and that these interactions were associated with adolescents' positive emotional well-being. As is acknowledged by the researcher, the participants were dual-earner, two-parent, mainly white and relatively affluent American families, so again the generalisability of the findings may be limited.

It is generally acknowledged that associations found between shared family mealtimes and adolescent outcomes in current studies are unable to determine a causal link (i.e. higher functioning and connected families may share more meals together). Nevertheless, shared family mealtimes are linked to better adolescent outcomes and it is likely that communication between family members is the key.

\subsubsection{Gender of parent differences}

Research findings indicate that adolescents may have an individuated relationship with one parent and not the other (Grotevant\& Cooper, 1986), and that the gender of the participants affects parent-adolescent communication (Flannery et al, 1993; Noller, 1995). Mothers tend to spend more time with adolescents than do fathers (e.g., Phares, Fields \&Kamboukos, 2009) and adolescents have perceptions of mothers 
as nurturing and fathers as authority figures, and these perceptions influence their expectations and perceptions about their relationships with their parents (Thornton, Orbuch \& Axinn, 1995). Adolescents perceive their mothers as more open to listening to them and tend to disclose more to mothers than to fathers. In addition, daughters as opposed to sons disclose more personal issues to mothers (Smetana, Metzger, Gettman \& Campione-Barr, 2006; Lila et al., 2006).

In an Italian study comparing parent-adolescent communication in foster, intercountry adoptive and biological families, Rosnati, Iafrate and Scabini (2007) found that mothers experienced more open communication with their adolescents than did fathers across all three family types. Although foster parents and children reported more difficulties in their communication than did other types of families, the authors acknowledge that this result is likely to be related to the previous negative experiences of the children that led to their being in foster care.

Adopted adolescents reported experiencing better communication with both parents than did adolescents living in either biological or foster families. On the other hand, adoptive parents' reports about their communication quality were similar to the reports of biological parents. In adoptive families, discrepancies were found between mothers' and adolescents' perceptions of their communication. Adolescents reported more open and less problematic mother-adolescent communication than did their mothers. On the other hand, adolescents and fathers' reports were more congruent.

\subsection{Transmission of Values}

A crucial function of parent-adolescent communication is the transmission of values that are important to that particular family and the culture and/or subculture in which the family is embedded. Family cultures are made up of values and family members' views about acceptable behaviours and ways of relating. Values may reflect views about such issues as gender roles, religion, sexuality, money, alcohol, the environment and a range of other issues. Parents strive to teach their children the values that are important to them (e.g. Lila et al., 2006). Communication is an important aspect of how these values are established and transmitted from parents to their children and and depends upon how motivated family members are to engage in discussions about values that are central to their beliefs (Pinquart \& Silbereisen, 2004). Such discussions between parents and adolescents play an important role in how parents pass on their beliefs and values to their adolescent.

In a study exploring the transmission of values around gender roles (Epstein \& Ward, 2011), a link was found between the values parents communicated about gender roles and the beliefs espoused by their adolescents. For example, there was a link between communication concerning body image and traditional gender beliefs. If young people perceived their parents as valuing idealised views of femininity and masculinity, they were more likely to favour traditional views of gender roles. 
It is generally accepted that gender roles are learned from multiple sources including mass media and peer groups as well as in the family through parents' modelling and the encouragement of particular activities (Blakemore \& Berenbaum, 2008; Ruble, Martin \&Berenbaum, 2006). In fact, this socialization continues throughout adolescence and emerging adulthood through interaction in the family, as well as in the larger society. Through family communication, values about the place of gender roles are reinforced (Martin, Ruble, \& Szkrybalo, 2002).

Flor and Knapp (2001) found that parents' religious behaviour and their desire for their children to be religious influenced their children's values around religion. Further, the more parents and adolescents engaged in discussions about religious beliefs, the more adolescents participated in religious behaviour (i.e., stated a belief in God and attended religious services) and the greater importance they placed upon religion. Although modelling and other aspects of adolescents' environments have an effect, this research suggests that the more discussions that parents have with their adolescents about their values and beliefs, where both parent and adolescent feel free to contribute, the more likely it is that their children will have similar values and beliefs.

Communication about sexuality is important to adolescents' development of sexual values and their approach to sexual health and risky sexual behaviours (Jerman \& Constantine, 2010). Parents who are knowledgeable about and comfortable with discussing sexual matters with their adolescents can have an impact on their children's sexual behaviour, such as postponing intercourse, using contraception and having fewer sexual partners (Dilorio, Pluhar \& Belcher, 2003).

Adolescents can also teach values to their parents. For example, Pinquart and Silbereisen (2004) found that adolescents passed on their values about the benefits of technology and the traditional way of life to their parents. These researchers also found that adolescents' religious values can be passed on to their parents. As might be expected, the influence of the adolescents on their parents' values was found to be stronger for families where parents were supportive and open to listening to the opinions of their adolescent.

\subsection{Decision-Making in Families with Adolescents}

As we have noted earlier, adolescents have expectations of a greater say in their family life and the decisions that affect them. During adolescence young people expect to make more of their own choices and participate more in family decision-making (Fallon \& Bowles, 1998). They usually look to their parents for help on important decisions such as educational and career choices and to their peers for help on issues such as what music to listen to, movies to see and books to read. Peers are also seen as more important for decisions such as hairstyles and clothing (Smetana, CampioneBarr \& Daddis, 2004). 
Because of the expectation that they should have more say in their own lives, adolescents may believe that lying to parents is fine if they think that they themselves are the ones who should make the decision (e.g., about going to a party; Jensen, Arnett, Feldman \& Cauffman, 2004). Parents may need to adapt to new ground rules about where their decision-making is appropriate and where they need to be flexible so that adolescents can over time develop their autonomy and their ability to make decisions on their own (Byrnes, 2002; Jensen et al., 2004;).

Adolescents whose parents tend to discuss decisions with them, and allow them to contribute to family decision-making, show greater positive adjustment, whereas adolescents whose parents allow them to make their own decisions unaided, and don't spend time encouraging and training them, tend to show poorer adjustment and more deviant behaviour (e.g. Goossens, 2006b; Van Petegem, Beyers, Vansteenkiste, \& Soenens, 2012). On the other hand, adolescents whose parents tend to control decision-making, without regard to their adolescents' views or input, and without explaining the reasons behind their decisions, are likely to grow up believing that communication is not important and have little confidence in their own decisionmaking abilities. Parents who encourage open discussion and value the opinions of their children teach them the importance of communication and encourage their young people to have confidence in their own decision-making abilities (Koerner \& Fitzpatrick, 2004).

Adolescents need to learn how to make good decisions by themselves as they become more involved in activities without parental supervision. Their ability to decide between decisions that are within their competence and those decisions about which they should seek advice is also an important part of learning to be autonomous (Byrnes, 2002).

The degree of control that adolescents and their parents think they should have varies depending on the issue. In a study involving African-American adolescents and their families it was found that for issues that were considered personal (such as what clothes to wear) the adolescents' contribution to decision-making was greater than that of mothers. For issues that involved conventional matters (such as using manners) and prudential issues (related to matters of risk) or decisions that might affect their futures (such as whether to have sex), the parents' contribution to decision-making was greater (Smetana et al., 2004). This study also showed that, over time, adolescents believed that more of the decisions were theirs to make. For example, the adolescents in this study believed that prudential and conventional issues should become joint decisions over time, whereas mothers continued to view them as parent decisions. Adolescents saw personal and multifaceted issues (e.g. how late to stay out at night) more as decisions for themselves, whereas mothers again viewed them as joint. Of course, age is likely to be important when parents consider decision-making. Seventeen-year-old adolescents are likely to have more say in such decisions than are 13-year-olds. 
Van Petegem and colleagues (2012) investigated autonomy through adolescent dependent versus independent decision-making and adolescents' motives for making decisions independently or with parents. These authors present independence, as measured by type of decision-making, and personal choice (self-endorsed) about parents' involvement as different ways of viewing autonomy. The two constructs are related and were found to have an impact on adolescent adjustment. As would be expected an absence of parental involvement in adolescent decision-making was related to increased behavioural problems; however, regardless of the level of parental involvement in decision-making the level of choice adolescents had in this involvement was positively related to adjustment. As previously mentioned, the degree of control that adolescents and their parents endorse varies across type of issue (Smetana, et al., 2004) and adolescents learning to decide between decisions that they make themselves and those in which they should seek parental support form an important part of learning to be autonomous (Byrnes, 2002).

Parenting style also affects the balance of power and decision-making in families with adolescents. In a study where both interview and questionnaire measures were used, Mackey and colleagues (Mackey, Arnold \& Pratt, 2001), explored the contribution of parenting styles to adolescents' seeking advice about decisions they believed were important (e.g., what subjects to take at school). Mackey et al. found that when parents had a more authoritative parenting style, and were involved and responsive, their adolescents were more likely to seek their advice. (See Chapter 3 for a more comprehensive discussion of parenting styles). Bednar and Fisher (2003) also looked at the impact of parenting practices on whether adolescents sought advice from their parents or their friends. Not surprisingly, the responsiveness of parents seems to be a key factor in whether adolescents will refer to them or to their peers when making decisions. According to Bednar and Fisher adolescents tend to refer to responsive parents over peers, and peers over controlling, permissive or neglectful parents when making important decisions.

For many young people, adolescence is a time when they need to make important career decisions. For this reason, parents need to know how they can assist their adolescents to make such decisions and to feel confident in the decisions they make. Parents need to show interest and provide support for their adolescents as they consider career options and deal with the complexity of this issue. Further, providing emotional support may be more important than just giving the young person specific information about careers (Keller \& Whiston, 2008).

In summary, adolescence is a time when young people expect more control in family decision-making, gain more opportunities for making the increasingly important decisions that affect their lives, and need to feel confident about those decisions. When parents are able to show their children that they are proud of them and have confidence in their ability to make these important decisions, their adolescents are likely to increase their decision-making abilities (Keller \& Whiston, 2008). Although adolescents generally value parents' opinions about the issues they 
are facing, there is evidence that adolescents are not always willing to talk about serious issues with their parents (Montemayor, 1983; Noller \& Callan, 1990), especially when they view the issue as a particularly personal one (Smetana, Metzger, Gettman \& Campione-Barr, 2006).

\subsection{Disclosure and Secrecy}

Parent-adolescent communication about risky behaviours can serve as a protective factor for adolescents (e.g. Guilamo-Ramos, Jaccard, Dittus \& Bouris, 2006). Parental monitoring and awareness of their adolescents' activities is associated with adaptive behaviour on the part of the adolescents. Parental monitoring of their adolescents has been associated with higher school performance and less externalising behaviour and substance use (e.g., Blocklin, Crouter, Updegraff \& McHale, 2011; Padilla-Walker, Harper \& Bean, 2011). However, parents mainly have knowledge of their adolescent's behaviours and activities through adolescents' direct disclosures (Kerr \& Stattin, 2000). These findings highlight once again the importance of the family climate being open and responsive, as well as the quality of the relationship and communication between parents and adolescents.

Parents tend to seek more information from daughters than sons about what is happening for them, and daughters also tend to offer more information about themselves to their parents than do sons (Kerr \& Stattin, 2000). Smetana and colleagues (2006) found that adolescents did not rate personal issues (e.g., how they spend their free time) as coming under parental authority, nor did they believe that they were obligated to disclose information about these issues to parents. These beliefs by adolescents about their obligations (or lack of) to tell their parents about themselves were associated with adolescents' reports of their actual disclosure to parents. Therefore adolescents are less likely to disclose to parents how they spend their free time and more likely to disclose issues around topics such as future education. Of course, it is important for adolescents to discuss issues such as education with their parents, since presumably they will need their parents' financial and emotional support for the decisions they make.

When adolescents perceive their parents as more trusting of them, they are more likely to disclose to their parents (Kerr, Stattin \& Trost, 1999). Guilamo-Ramos and colleagues (2006) investigated the links between adolescents' perceptions of their mothers' expertise, trustworthiness and accessibility, the frequency of their discussions with their mothers about risky behaviours and their reports of their actual behaviour. This study focused specifically on sexual behaviour and smoking. These researchers found that adolescents' perceptions of mothers were associated with the frequency of the communication between them and their mothers, which in turn was associated with adolescents' behaviours. When adolescents viewed their 
mothers more positively they were more likely to discuss issues with them, and less likely to engage in risky behaviour.

Keeping secrets from parents is quite different from not disclosing to parents as keeping secrets requires careful monitoring of what is said (Finkenauer, Engels \& Meeus, 2002). Finkenauer and colleagues (2002) found that keeping secrets from parents contributed to adolescents' feelings of autonomy, although at the cost of their psychosocial well-being. Adolescents who kept secrets from parents were more likely than other adolescents to report physical complaints and depressive mood. A Dutch study (Hawk, Keijsers, Frijns, Hale, Branje, \& Meeus, 2013) found that the more adolescents perceived their parents' to be invasive (i.e. wanting to know everything about them), the more likely the adolescents were to report keeping secrets from their parents. Therefore, as parents try to increase the information they have about their adolescent, depending upon how they go about it, they may in fact be achieving quite the reverse outcome.

Smetana et al. (2006) unexpectedly found a positive relationship between adolescents' disclosure to parents and the level of psychological control used by parents, suggesting that adolescents with parents who are psychologically controlling "may feel subtly coerced to disclose personal issues" (p.213). These authors argue that further investigation of this issue is required, and they suggest that some types of disclosure by adolescents to parents may be helpful whereas other types may not. Clearly, more research is needed to explore this issue.

\subsection{Conflict in Families with Adolescents}

During adolescence, the quality of communication between parent and child is likely to decrease, and conflict is likely to increase (Montemayor, 1983; Sroufe et al., 2005; Steinberg, 1987). Conflict generally increases from early adolescence, tends to peak at mid adolescence (De Goede, 2009; Jensen-Campbell and Graziano 2001) and usually decreases again in late adolescence (Montemayor, 1983; Steinberg \& Morris, 2001). Conflict is not necessarily a bad process and will occur in all intimate relationships and most of the conflict between parents and adolescents involves everyday matters such as household tasks (Adams \& Laursen, 2001; Rubenstein \& Feldman, 1993), and parent-adolescent relationships usually remain, for the most part, positive and close (Steinberg \& Silk, 2002).

Conflict between parents and adolescents is important as part of the renegotiation of roles and the adolescents' development of autonomy (Collins \& Laursen, 2004). In addition, adolescents learn how to deal with conflict within their family interactions (e.g. Holmes, Bond \& Byrne, 2012; Parke \& Buriel, 2006). Conflict can positively assist with the development of relationships, adolescents' problem-solving skills, and contribute to adolescents' personal and cognitive development (Holmes, Bond \& Byrne, 2012). 


\subsubsection{Identity Styles and Conflict}

As we explained in Chapter 2, identity styles (Berzonsky1989, 1992) are associated with the styles parents use in socialising their children. In addition, the ways that parents and their adolescents deal with conflict are also associated with their identity styles (Missotten, Luyckx, Branje, Van Halst \& Goossens, 2011). This Belgian study explored the links between mother-adolescent conflict and identity formation. Adolescents who tended to use an informational style had fewer conflicts with their mothers and were more positive in their approaches to conflict when it occurred. Presumably this finding was due to their more flexible approach to issues than those with other identity styles.

In this same study by Missotten and colleagues (2011), those using a diffuseavoidant style had more conflict with their mothers and were more likely than those with other styles to withdraw from conflict situations, leaving many conflicts unresolved. According to these researchers, when these young people did get involved in the discussions, they were more likely than the other styles to behave aggressively and because of their compromised ability to see their mother's perspective, they had difficulty negotiating a positive solution.

Having a normative style was not associated with the frequency of conflict with mothers, but adolescents using a normative style tended to be compliant when they did face a conflict. These adolescents, when confronted with authoritarian parenting, tend to do as they are told. Although this is a very interesting study, one weakness was the focus on mothers only, so we have no idea how they react when in conflict with their fathers.

\subsubsection{Gender of Adolescent Differences in Parent-Adolescent Conflict}

Whereas some studies have found gender of adolescent differences in parentadolescent conflict (Noller \& Callan, 1990), other studies have found few gender differences (Caughlin \& Ramey, 2005; Wierson, Armistead, Forehand, McCombsThomas \& Fauber, 1990). Allison and Schulz (2004) found no gender differences for the frequency of parent-adolescent conflict. However, they did find more intense conflict between parents and adolescent daughters, as opposed to sons, about topics such as household tasks, personal appearance, and personal autonomy. In this study the only topics that generated more parent-son conflict, as compared to parentdaughter conflict, were doing homework and achieving at school. These findings were discussed in terms of gender stereotyping and conceptions of femininity and masculinity. These findings are not surprising given the gender expectation that daughters perform more household tasks and pay more attention to their appearance than sons. 
Honess and colleagues (1997) found that more frustration and less escalation in discussions with parents was reported by daughters as opposed to sons. The authors suggest that this result is consistent with notions about the different experiences of girls and boys, whereby girls are taught to value both closeness and autonomy whereas boys, mainly seeking autonomy, would escalate the conflict to achieve their desired outcome. In line with this reasoning, it has been found that adolescents are likely to see lying to parents as acceptable when they perceive the situation as one that is up to them and that boys tend to see lying as more acceptable than do girls (Jensen et al., 2004).

\subsubsection{Conflict Styles}

It has long been suggested that those families that find their children's transition to adolescence difficult may differ from families that experience a relatively easy transition, in either the content of their conflicts or the ways they deal with those conflicts (Gehring, Wentzel, Feldman, \& Munson, 1990). Several conflict styles have been identified as used by parents and adolescents. These styles are: coercion (both parties making demands of each other), parent-demands/adolescent withdraws (parent making demands and the adolescent avoiding the discussion), adolescentdemands/parent-withdraws (adolescent making demands and the parent avoiding the discussion) and mutuality (parent and adolescent problem-solving together; Noller, Atkin, Feeney \& Peterson, 2006).

Coercive interactions between parents and adolescents have been linked to adolescent adjustment and in particular to the development and establishment of aggressive behaviour (e.g. Patterson, 1982; Granic \& Patterson, 2006). Patterson (e.g., 1982) has been influential in the establishment of the links between coercive parentchild practices and the subsequent development of aggressive behaviours in children. A pattern of mutual coercion is said to intensify and escalate over the period of the child's development. The child learns to respond coercively to demands and does not learn to cope with frustration in a constructive way.

Demand/withdraw has been widely studied in marital relationships (e.g. Christensen, 1988; Christensen \& Heavey, 1990; Noller, Feeney, Roberts \& Christensen, 2005; Noller \& Roberts, 2002). Recently, however, researchers have investigated the use of demand/withdraw in parent-adolescent conflict (Caughlin \& Ramey, 2005; Caughlin \& Malis, 2004a; Caughlin \& Malis, 2004b;). Caughlin and Ramey (2005) found that demand/withdraw in parent-adolescent relationships followed a pattern similar to that found in marital relationships, whereby one person's demanding was associated with the other persons' withdrawing. As is true for married couples who use demand/withdraw patterns of interaction (Eldridge \& Christensen, 2002), Caughlin and Malis (2004a) found that the more parents and adolescents engaged in demand/ withdraw conflict, the less satisfied both interactants were with their relationship. 
Van Doorn, Branje and Meeus (2011) in a 4-year longitudinal Dutch study, found that the conflict resolution styles of parents and adolescents changed over time and that adolescents increased their use of avoidance or withdrawal with both parents from early to mid-adolescence but only temporarily with their mothers. Further, the mothers in this study did not report any increase in their use of withdrawal as a response to conflict with their adolescent as opposed to fathers who indicated that they did increase their use of this response. The authors indicate that this finding may be due to the fact that mothers spend more time with their adolescents. It seems likely therefore that mothers need to adapt their approach to conflict resolution with their adolescents more quickly than do fathers. In addition, there is evidence that men tend to withdraw from conflict, at least in the marital relationship, more often than women do (Noller et al., 2005).

Mutuality is expressing one's own opinion and respecting the beliefs or ideas of the other, and being able to negotiate the two views to seek resolution. Children's exposure to positive conflict interactions can be an important means of learning how to deal with conflict (Grych \& Fincham, 1993). Boys who utilise a mutual or compromising approach to conflict have been found to do better on a range of adjustment measures such as higher academic achievement and lower levels of poor conduct and depression (Rubenstein \& Feldman, 1993; see Table 4.1)

Table 4.1: Conflict styles and outcomes

\begin{tabular}{|c|c|c|}
\hline Style & Description & Outcome \\
\hline Coercive & $\begin{array}{l}\text { Both parties behave aggressively and } \\
\text { make demands of each other }\end{array}$ & $\begin{array}{l}\text { Problems with psychological adjustment } \\
\text { of adolescent; } \\
\text { development of aggressive behaviour in } \\
\text { adolescent }\end{array}$ \\
\hline $\begin{array}{l}\text { Demand/ } \\
\text { withdraw }\end{array}$ & $\begin{array}{l}\text { Either adolescent or parent makes } \\
\text { aggressive demands and the other party } \\
\text { withdraws or avoids dealing with the } \\
\text { conflict }\end{array}$ & $\begin{array}{l}\text { Less satisfaction in relationship between } \\
\text { parent and adolescent }\end{array}$ \\
\hline Mutual & $\begin{array}{l}\text { Both parties express own opinion at the } \\
\text { same time as showing respect for opini- } \\
\text { ons of the other } \\
\text { Both parties willing to negotiate }\end{array}$ & $\begin{array}{l}\text { Adolescent learns positive ways of } \\
\text { dealing with conflict } \\
\text { Adolescent's feelings of well-being } \\
\text { strengthened } \\
\text { Positive adjustment for adolescent }\end{array}$ \\
\hline
\end{tabular}

In general, research shows that higher quality parent-adolescent communication is related to multiple indicators of adolescent well-being. Specifically, positive parentadolescent communication during conflict is related to higher self-esteem, fewer depressive symptoms, less self-harm, and greater life satisfaction (Ackard, NeumarkSztainer, Story, \& Perry, 2006; Jackson, Bijstra, Oostra, \& Bosma, 1998; Tulloch, 
Blizzard, \& Pinkus, 1997). Of course it is possible that adolescents with higher selfesteem are more likely to engage in positive communication with their parents, in any case.

Grotevant and Cooper (1986) indicate that adolescents' experiences of supportive behaviour from their parents, particularly whilst they are exploring their identities, has a positive impact on their psychosocial development. Allowing adolescents their own views, while at the same time challenging differing ideas, gives them confidence in their opinions and facilitates their development and their ability to reflect on the views of others in comparison to their own (Sandy \& Cochran, 2000). As adolescents develop they and their parents change their conflict resolution styles and increase their use of positive problem solving in ways that reflect more equality in their relationships (Van Doorn et al., 2011). These types of findings support the proposition that adolescents learn their communication and relating skills from their interactions with their parents and highlight the importance of conflict and how it is approached for adolescent skill development. It is important to note that whereas this study indicated increased maturity in response to conflict for older adolescents, other researchers (e.g., Tucker, McHale \&Crouter, 2003) have found less constructive conflict occurring between older adolescents and their parents.

\subsubsection{Positive Aspects of Parent-Adolescent Conflict}

Conflict with parents is not necessarily a negative experience for adolescents and does not inevitably harm the parent-adolescent relationship (Scholte \& Van Aken, 2006). To a degree, conflict with parents may be healthy for the personal development of adolescents (Goosens, 2006b) as they learn to assert themselves and make their needs and preferences known. After all, everyone needs to learn to deal with conflict constructively, a skill that is likely to be useful throughout one's life and across all kinds of relationships.

Another function of parent-adolescent conflict may be to facilitate the renegotiation of roles and rules, and to establish a different power balance with parents in order to develop more adult-adult relationships (Collins \& Laursen, 2004; Collins \& Steinberg, 2006; Grotevant\& Cooper, 1986). Such conflict may be a forum in which, within a supportive family environment, adolescents can develop autonomous thought and advance their psychosocial development (e.g. Rubenstein \& Feldman, 1993).

Adolescents learn their conflict behaviours, and how to cope with and respond to conflict, from their interactions with their parents. There is evidence that the types of conflict patterns that parents engage in influence the conflict patterns between parent and child (Noller, Feeney, Peterson \& Sheehan, 1995; Van Doorn, Branje \& Meeus, 2007). For example, when parents use demand-withdraw (one person making 
demands while the other avoids the discussion) as a conflict style, their children are also more likely to deal with conflict in this way (Caughlin \& Malis, 2004b; Noller et al., 1995; See Table 4.2)

Table 4.2: Positive and Negative Aspects of Conflict

\begin{tabular}{ll}
\hline Positive aspects & $\begin{array}{l}\text { Can help adolescent develop and express autonomous thought } \\
\text { Facilitates renegotiation of roles and rules toward more mature relationships } \\
\text { with parents } \\
\text { Adolescent learns how to cope with and resolve conflict }\end{array}$ \\
\hline Negative aspects & $\begin{array}{l}\text { Possible decrease in warmth in parent-adolescent relationship } \\
\text { Likely increase in parent's attempts at maintaining control of adolescent } \\
\text { Parent may devalue adolescent's ideas and impede development of indepen- } \\
\text { dent thought } \\
\text { If adolescent very angry, may become involved in problem behaviour (drugs, } \\
\text { delinquency etc.) }\end{array}$
\end{tabular}

\subsubsection{Negative Aspects of Parent-Adolescent Conflict}

The adolescent's striving for autonomy and individuation from the family of origin can create an increase in conflict in the parent-adolescent relationship (Callan \& Noller, 1986; Collins \& Laursen, 2004; Flannery et al, 1993; Montemayor, 1983; Steinberg, 1990). Parent-adolescent conflict is related to a decrease in warmth in the relationship, and an increase in parents' attempts at maintaining control, as the adolescent strives for autonomy (Flannery et al, 1993; Steinberg, 1990). An important aspect of developing autonomy is to feel free to disagree with one's parents and to react differently from them in family situations (Goossens, 2006b; see Chapter 2 for more discussion of autonomy and individuation).

Several authors suggest that the processes involved in parent-adolescent conflict may have specific effects on adolescent adjustment. For example, Hauser, Powers and Noam (1991) argue that some parents may be distressed by disagreements with their adolescent, and may respond by withdrawing or devaluing the adolescent's opinions, which can hinder the adolescent's development. McCombs, Forehand, and Smith (1988) found that fewer problem-solving skills were observed in adolescents who had mothers who were avoidant of conflict. Such findings indicate the importance of developing an understanding of the processes involved in parentadolescent conflict and imply that the ability of families to support their adolescents' differing views could have an impact on adolescent adjustment. High levels of parentadolescent conflict have been associated with a range of adolescent problems, such 
as school difficulties, early sexual activity and pregnancy, depression, and physical abuse (e.g. Noller, 2005a). Also problems of low self-esteem and drug use appear to be related to high levels of adolescent-parent conflict (Caughlin \& Malis, 2004; Montemayor, 1983; see Chapter 6 for further discussion of adolescent issues and problem behaviours).

Many studies have found that destructive family conflict is associated with delinquency in adolescence (e.g. Sigfusdottir, Farkas \& Silver, 2004). Based on Agnew's (1992) general strain theory, Sigfusdottir and colleagues investigated whether the association between aversive family conflict and delinquency was related to adolescents' subsequent feelings of anger and depressed mood. General strain theory proposes that strain in the form of negative life events (in this study defined as negative family conflict), is positively related to delinquency. These researchers found that the level of family conflict particularly increases the likelihood of adolescents becoming involved in delinquent behaviour when the adolescents are very angry. Thus, when adolescents reported more negative conflict in the home, they reported more feelings of anger, which in turn were associated with more self-reports of delinquent behaviour. (See Table 4.2)

\subsection{Parental discipline}

Elliot and Feldman (1990) refer to adolescence as a time for self-discovery and for the acquisition of skills that are needed for adulthood. Adolescents are developing new cognitive abilities that can change the ways in which they view themselves and their parents (Goossens, 2006a; Larson \& Richards, 1994). For example, as mentioned in Chapter 2, adolescents have generally developed formal operational skills, allowing them to reflect upon and question existing family conventions (Grotevant \& Cooper, 1986). These advanced reasoning skills mean that adolescents see parental regulation differently from the way they saw it earlier in their development. At this stage of their lives, they are more likely to argue about the relevance of parental rules and values to themselves than when they were younger.

Smollar and Youniss (1989) investigated the connections between adolescents' perceptions of their relationships with their parents and the concept of individuation discussed in the previous chapter. These researchers used adolescents' beliefs about their parents' "all-knowingness" and right to wield authority as a measure of individuation and found that the 15-16 year old adolescents were in a process of 'de-idealising' their parents, and were developing a greater sense of the importance of their own opinions. They also found that the older adolescents participating in the study (17-23 years) did not generally appear to be still in a process of 'de-idealising' mothers, but had come to recognise their mothers as individuals.

Interestingly, for these older adolescents 'de-idealisation' of fathers was ongoing, and a perception of fathers as individuals, rather than as authority figures, was not 
evident. This finding is interesting given that mothers are often more involved and responsible for the discipline of their adolescents than are fathers (Phares et al., 2009). These results are the likely outcome of mothers and adolescents both having more time to renegotiate their relationships and a more urgent need for doing so in order to relate in a relatively harmonious manner. In other words, mother-adolescent relationships may mature earlier than father adolescent relationships (Van Doorn et al., 2011) but it seems likely that this process occurs over a longer time period with fathers.

Smetana (1988) examined parents' and adolescents' perceptions of the areas of adolescent behaviour that come under parental authority. She suggests that parentadolescent conflict is due to the differing perceptions of parents and adolescents about the aspects of the adolescents' lives over which the parents may exercise authority. The situations were categorised into four domains; moral (e.g. stealing), conventional (e.g. not cleaning up after a party), personal (e.g. sleeping late on the weekend) and multifaceted (e.g. dressing in punk clothes). These issues are strongly related to adolescents' concepts of their identity and values. The families participating in this study were predominantly middle-class, Caucasian families that included a child aged from 11 to 17 years. All family members viewed both moral and conventional issues as being more appropriately under parental authority than personal issues; however, mothers and fathers were more likely to view personal issues as under parental authority than were adolescents.

Parents, who allow their adolescents independence whilst providing support, assist them to develop confidence in their own abilities whereas those that are overly controlling can inhibit their adolescents' confidence in their abilities to self-regulate and make decisions (see previous section on decision-making). Of course parents need to maintain guidelines and monitoring during adolescence as well as allowing their adolescents to experience the consequences of their bad behaviour where practicable. Padilla-Walker (2008) reported similar findings to those of Smetana (1988), despite the fact that her study was conducted 20 years later than Smetana's study. Padilla-Walker found that mothers tended to be more angry and directive in their use of discipline in situations where their child had done something dangerous (e.g. drinking or smoking) than if they had broken a conventional rule (e.g., come home late). The adolescents in this study also indicated that mothers were more likely to discipline them when they broke a conventional rule than a personal one (e.g. wore clothing not approved of). Mothers' discipline tended to be accepted for behaviours that were dangerous or related to moral issues (e.g. cheating on a test), but not for conventional or personal issues. The adolescents also indicated that the best response for mothers to make was to talk to their adolescents and reason with them.

If parents engage with their adolescents about how the behaviour impacts on them and seek the adolescents' involvement in the process (i.e., deciding what the consequence should be) adolescents may feel better about the situation and 
their relationship with their parents (DeRoma, Lassiter \& Davis, 2004). Further, such engagement with adolescents assists with their self-esteem and gives them an opportunity to learn to regulate their own behaviour (De Roma et al.), which is a major goal of parenting during the adolescent years.

\subsection{Communication with Siblings}

As noted earlier, sibling relationships are important and the longest relationship that most people have. In relationships characterised by warmth, siblings reported high levels of self-disclosure and emotional understanding between them (Howe, AquanAssee, Bukowski, Leboux \& Rinaldi, 2001). In addition, siblings were more likely to self-disclose to each other when they held a degree of trust.

According to Bedford and Volling (2004) interactions between siblings can either promote or undermine healthy development depending on the extent to which they encourage strategies of emotional self-regulation (being able to control one's own emotional reactions). Specifically, they can assist each other with the development of emotional self-regulation by providing each other with opportunities to express emotions openly and in socially acceptable ways.

During adolescence the warmth of the parent-adolescent relationship is likely to decline because of the increased time young people spend with their peers, and particularly as they get older siblings can be an important support (Dunn, 1992; Noller, 2005b). More sibling conflict has been reported in families where there were high levels of conflict between the parents (Noller, 2005b). Sibling conflict may increase in early adolescence (e.g., Brody, Stoneman \& McCoy, 1994) as they come into conflict over issues such as household duties, possessions and behaviour toward each other (Roloff \& Miller, 2006). The level of conflict in sibling relationships can be greater than in any other relationship in which adolescents are involved, including relationships with mothers and fathers (Lila et al, 2006).

Parents' responses to their children's arguments can have an impact on sibling relationships. For example, if parents side with the weaker sibling or impose punishments for sibling conflict, this interference can result in further conflict and negative feelings between the siblings; on the other hand, parents advising or explaining feelings as a response to the conflict may not impact negatively on the relationship between siblings (Roloff \& Miller, 2006). Therefore parents' responses to conflict between their children are an important aspect of family relationships and the general family climate.

Although sibling conflict is related to higher levels of anxiety, depression and maladaptive behaviour in adolescents (Stocker, Burwell \& Briggs, 2002), sibling relationships can be of benefit to adolescents. Siblings can be a valuable support to each other in adolescence (e.g., Jenkins-Tucker, McHale \&Crouter, 2001; Lila et. al, 
2006), perhaps especially in times of conflict between the parents (Noller et al., 2008; Sheehan et al., 2004).

Jenkins-Tucker and colleagues (2001) found that the ways siblings support one another varies across contexts. Within the home, siblings tended to support each other in a more egalitarian manner, whereas in social situations, or situations outside of the family, older siblings' support of younger ones was more common. Older siblings were found to provide support in the form of advice to younger siblings on social life, schoolwork and risky behaviour; this finding was more marked for siblings from small families. It was also found that younger to older sibling support about risky behaviours was a factor in sibling conflict. Older siblings seem not to appreciate being told what to do by a younger sibling.

A positive association was also found between level of parent-adolescent conflict and adolescent support for both older and younger siblings. The authors suggest that siblings may become allies when one or both of them are experiencing severe conflict with parents. On the other hand, where parents treat one sibling more favourably than the other, siblings are more likely to have a conflicted relationship and are less likely to be allies (Noller, 2005b; Sheehan \& Noller, 2002). Even if parents are perceived to respond more favourably to one sibling than the other, problems are likely to arise in family relationships. (See Chapter 5 and Sheehan et al. (2004) for more discussion of sibling relationships in divorcing families). Older siblings from single-parent families are likely to take on a caregiver role and to act more like parents than siblings (Sheehan et al., 2004; Zukow-Goldring, 2002), and this situation may have a disruptive impact on the sibling relationship.

There is little research about the differences in sibling communication across family types, although one study explored relations between affection and hostility in adolescent sibling relationships and adolescent behavioural outcomes (Padilla-Walker, Harper \& Jensen, 2010). These researchers found that sibling affection was positively related to self-regulation and prosocial behaviours and negatively related to externalizing behaviours (acting out and blaming others) for both family types. Hostility in the sibling relationship on the other hand was positively related to externalizing behaviours and having a sister was negatively related to internalizing behaviours (such as depression and anxiety). In other words, affectionate sibling relationships had a positive effect on adolescent behaviour whereas hostile sibling relationships tended to have a negative effect on adolescent behaviour and having a sister lessens the likelihood of internalizing problems. It is important to note that associations were stronger for adolescents from two-parent families than for those from single-parent families. With regard to this study it is important to acknowledge that this study was based on selfreports of a single sibling from the two types of families. As the authors note, ecological validity would be increased if reports were obtained from both siblings. 


\subsection{Family Relationships and Relationships with Peers}

During adolescence, intimacy in peer relationships becomes more a feature of friendships than is true for younger children. These relationships are important because peer relationships have been shown to be significant in the development of social competence (Adams \& Laursen, 2001). Whereas relationship skills are initially developed in the family, later peer relationships are linked with these early learnings. Based on what they have learned in the family, adolescents practise and develop their relationship skills within their peer relationships (Collins \& Steinberg, 2006; Sroufe et al., 2005) and the development of relating skills learned with peers can influence the relating styles used with parents (Van Doorn, Branje et al.,2011).

More positive peer relationships have been found for adolescents who have competent social skills, developed in warm and supportive family environments (Lieberman, Doyle \& Markiewicz, 1999). Knoester and colleagues (2006) found that adolescents, whose parents had good relationships with them and who provided plenty of supervision, were more likely to choose friends who were pro-social and less likely to choose friends with problem behaviours. They suggest that the focus of research should move from whether parents or peers have more influence on adolescents to investigating the connections between the two sets of relationships and the ways peer relationships are formed.

Adams and Laursen (2001) investigated the differences between adolescents' conflict with parents and their conflict with friends. They found that the dynamics of these conflicts were consistent with the relative power balances that adolescents enjoy with friends as opposed to parents. Parent-adolescent conflict was found to contain more power assertion than conflict between adolescents and their friends. The two relationships involve quite different dynamics. Adolescents' relationships with their parents tend to be more unilateral than peer relationships and continue regardless of daily disputes. Due to the experience of such equality in peer relationships, adolescents strive to gain more equality in relationships with their parents (e.g. Adams \& Laursen, 2001; Youniss \& Smollar, 1985). When dealing with relationships with friends, however, adolescents need to be mindful of not doing anything that may damage those relationships if they want those relationships to last. Just as peer relationships influence adolescent-parent relationships, adolescentparent relationships also influence the peers with whom adolescents form friendships.

Parent-child relationships and parental supervision are associated with adolescents' friendships with peers (Knoester, Haynie \& Stephens, 2006). Parents' prosocial behaviour, responsiveness and positive communication with their adolescents are associated with positive qualities in adolescent-peer relationships (Cui, Conger, Bryant \& Elder, 2002). Also, parents' direct involvement in their adolescents' peer relationships, such as chauffeuring and discussing peer relationships, has been found to promote positive adolescent-peer relationships (Updegraff, McHale, Crouter \& Kupanoff, 2001). 
Updegraff and colleagues (2001) found that parents' direct involvement with their adolescents' peers was more central for boys than for girls. Mothers' involvement with these peers was associated with the time sons spent with a best friend, and fathers' involvement was associated with their adolescents' participation with their peer group and the emotional qualities of their friendships. Mothers' association with daughters' friendships was linked with more time spent with friends but not to the emotional quality of their friendships. The authors suggest that girls may more readily develop intimate relationships than boys, and therefore promoting opportunities for peer contact, such as by chauffeuring or allowing sleep-overs is more important for girls than is likely to be the case for boys.

\subsection{Technology and Communication}

In the current decade most families live with many devices that provide them with media information and input. It is quite common for adolescents to have their own mobile phones (e.g. Madell \& Muncer, 2004), and access to computers, television sets, iPods and CD players or DVD players in their bedrooms (e.g., Jennings \& Wartella, 2004; Wilson, 2004), providing more opportunities for them to be isolated from the rest of the family.

Adolescents' use of technology in a private space, such as the bedroom, can be seen as problematic due to the inability of parents to monitor what they are watching and doing. Having a computer in their bedroom, where their parents cannot easily monitor what they are doing, has been found to predict adolescents' online aggression (Law, Shapka \& Olson, 2010). As previously mentioned, parents' ability to monitor their children's activities is important to their development and overall well-being (e.g. Kerr \&Stattin, 2000). Parents seem to be concerned about both the content of what children are seeing and how they can monitor and have a say in their children's use of technology (Jennings \& Wartella, 2004). However, the current trend is for adolescents to have more freedom and self-directed activity than has previously been the case (Bassi \& DelleFave, 2004).

Both positive and negative impacts of the use of technology have been reported. The use of technology may benefit adolescents in terms of their developing cognitive skills, improving their performance at school as well as their communication with family and friends (Punamaki, Wallenius, Nyugard, Saarni \& Rimpela, 2007). If families watch television together, this activity can encourage conversation. On the other hand, having the television on during meal times can inhibit table conversation (Wilson, 2004).

The negative impact of internet usage on friendships has changed because many adolescents now use the internet to maintain friendships through socialnetworking sites such as Facebook. These sites can provide more opportunities for connecting and communicating with friends rather than with strangers (Valkenburg 
\& Peter, 2009). Nevertheless, it is important for parents to inform their children of the dangers of sharing personal information and/or making arrangements to meet new acquaintances they know only from the internet. For example, in Australia police continually warn about the devious methods used by paedophiles to make new contacts.

Girls have been found to be more likely than boys to use a mobile phone and boys have been found to be more likely to use the internet than girls (Madell \& Muncer, 2004; Punamaki, et al. 2007). Boys tend to use modern technology to have fun and explore information, whilst girls tend to use technology for relationships and communication (Punamaki, et al. 2007). However, social networking sites may benefit boys more than girls, because adolescent boys are likely to find the internet an easier way to give personal information than face to face. These technologies can help them develop closer relationships with friends (Schouten, Valkenburg \& Peter, 2007). Also adolescents who tend not to use social networks on the internet have fewer friends in general than those who do (Bryant, Sanders-Jackson \& Smallwood, 2006), although it may be important to assess the quality and closeness of those relationships.

Adolescents use mobile phones mainly for making and receiving calls and text messaging (Madell \& Muncer, 2004). Mobile phones can be useful for keeping in contact with family members, including parents. Mobile phones have been found to be associated with family connectedness; parents and adolescents' increased use of their mobile phones to communicate was related to increased reports of family closeness (Padilla-Walker, Coyne and Fraser, 2012). Also parents can use this technology to monitor their adolescents, but the usefulness of the technology for such parenting purposes may not be reliable as adolescents can simply not answer their parents' calls (Ling, 2004), or not be open about what they are doing or where they are. In addition, parents may also engage in micro-managing of their adolescents, calling them and having them call in frequently to report what they are doing. This behaviour could compromise the young person's ability to develop the skills necessary to act autonomously. Such behaviour on the part of parents could also be seen as being overprotection that has been found to increase the probability of depression because of the messages of inadequacy that are central to overprotection (Parker, 1994). Therefore, the use of mobile phones for positive and shared communication between family members is helpful for family relationships but if this communication is intrusive for the adolescent then the impact may hinder relationships (PadillaWalker et al., 2012).

Weisskirch (2009) investigated the use of mobile phones as a parenting and communication tool. This study looked at mobile phone use in 196 parent-adolescent dyads and found no differences for age of adolescent (13-19 years), gender of either adolescent or parent, or cultural background (Euro American, Asian American, Latino, African American, and American Indian). It should be noted that the majority of the participants were Euro American ( $83 \%$ of the parents and $77 \%$ of the adolescents) and 
mother-adolescent dyads (76\%). This situation would have limited the ability to find differences.

The general findings indicated that the purpose and the emotional climate of the calls were linked to the quality of relationships and communication as well as parent and adolescent well-being. For example, parents using phone calls for monitoring and checking on school-work was associated with greater conflict, though higher self-esteem for parents. These parents are likely to gain esteem through a belief that they are performing their roles as parents, even though it seems likely from these data that they are alienating their young people. These findings are consistent with notions of adolescents seeking autonomy, and parents wanting to maintain a parenting role.

Also, parents calling their adolescent when they themselves were upset was associated with lower self-esteem for both parents and adolescents. On the other hand, greater self-esteem for parents and adolescents was associated with adolescents calling their parents for support. This finding is likely to be a result of parents feeling needed by their adolescents and close to them and adolescents experiencing their parents as available and caring. These results indicate that parent-adolescent mobile phone use reflects what is known about face-to-face communication, but that it can also enhance parent and adolescent well-being and relationships when the conversations are calm and supportive. Weisskirch (2009) points out that the immediacy of parental emotional support via mobile phone may be an important positive attribute of such communication.

Mobile phone use may also have a detrimental effect on adolescents. For example, in a study in Belgium, adolescents who were woken during sleep by text messages reported being more tired generally, at school and after the weekend (Van den Bulck, 2003). In a Finnish study, Punamaki and colleagues (2007) found that girls' mobile phone use and boys' computer use could negatively impact their health through poor sleep and subsequent tiredness. Some parents try to deal with the problem by having their teenagers give up their mobile phones at bedtime. Again, the success of such an arrangement is likely to depend on the quality of the relationship between parent and child and the extent to which the decision has been made in mutual discussion rather than enforced.

Another cause for concern is that technology is increasingly being used by adolescents for the purpose of bullying others, although face-to-face bullying is still more common (Hertz \& David-Ferdon, 2008). The use of technology for aggression has been labelled internet bullying, internet harassment and cyber-bullying. In a review of research about cyber-bullying, Hertz and David-Ferdon indicate that in this early stage of research there is evidence that making rude or nasty comments is the most common bullying behaviour and that instant messaging seems to be the most common technology used.

When being bullied using technology, victims may not know the perpetrator, although the research evidence suggests that such messages often come from 
someone the victim knows, and even a sibling. It is difficult for adolescents to address this type of bullying and of course it invades their private domains as well as the public domain. The Hertz \& David-Ferdon (2008) study indicates that as yet little is known about the impacts of cyber bullying; however, adolescents who are the victims are more likely to use alcohol and other drugs, have problems at school and be the victims of face-to-face bullying than those adolescents not being victimised.

Of course, it is difficult to assume a causal link between being bullied and drinking and drug use as there are likely to be many intervening factors contributing to both. These issues can impact on the family and many parents may not know how to assist their adolescent to cope with such issues. An extreme example of a parent demonstrating little knowledge of how to appropriately support their adolescent through such bullying occurred recently in a country town in Australia. A mother was convicted, and spent time in gaol, for the assault of an adolescent who was using the internet to bully her daughter. Clearly, this situation highlights the potentially negative consequences for whole families and the need for some constructive information as to how to respond to cyber-bullying.

Modern technology and media also have an impact on what adolescents are exposed to and how, for example, they currently learn about sexual attitudes and behaviour (Brown \& L'Engle, 2009; Epstein \& Ward, 2007; Hust, Brown \& L'Engle, 2008). These sources of sexual information are problematic because they rarely contain sexual health messages (Hust et al., 2008), nor do they encourage adolescents to think carefully before engaging in sexual activity. In these times, adolescents can view sexually explicit material quite easily and through a number of technologies, such as television, the internet and even their mobile phones (Roberts \& Foehr, 2004). While it is normal for adolescents to be curious about sexual behaviour as they mature physically, concerns exist around how viewing sexually-explicit material can mould their values and ideas about sexual relationships and behaviour (Brown \& L'Engle, 2009; Nathan, 2007). Boys have been found to view sexually explicit material more than girls (Brown \& L'Engle) and to learn more about sexual behaviour from the media and peers than from their parents (Epstein \& Ward, 2007). Developing an ability to relate sexually through these means is concerning and may create the impression that sexual behaviour cannot be controlled (see Chapter 6 for more discussion about adolescent sexual behaviour),

Parents' engagement and communication with adolescents about sexual behaviour can be important in monitoring the impact that exposure to sexual information and images may have. When parents communicate with their adolescents about sexual behaviour and watch television shows with them, even at low levels, it has been found that adolescents are less likely to engage in sexual intercourse at a young age (Bersamin, Todd, Fisher, Hill, Grube \& Walker, 2008). 


\subsection{Culture and Communication}

Literature about cultural influences and differences in family communication is scarce although many researchers acknowledge the importance of examining the impact of culture on family communication (Turner \& West, 2011). To examine all aspects of the many cultures that exist is a large task in itself without the necessity to look at the impact of the often out-of-step enculturation between parents and children of immigrant and refugee families.

We have mentioned previously "family culture" and the importance of shared values and understandings and the ways in which communication is an important aspect of creating family culture. Two broad cultural differences that are often discussed are individualism (where the culture encourages individual achievement and thoughts) and collectivism (where the culture encourages group achievement and cooperation). Western societies are considered to be increasingly individualist and Eastern societies are considered to be traditionally collectivist. Individualist cultures tend to promote open communication and discussion of values and collectivist cultures value less direct communication and rely on an embedded understanding of values. These cultural differences may provide challenges to families moving between multiple cultures.

Children tend to learn new languages more readily than their parents (e.g. PeaseAlvarez, 2002) and this can be at the cost of fluency in their parents' language (Hakuta, 2001). Also children have been found to acculturate more rapidly and to a greater degree than their parents (e.g. Pasch, Derdorff, Tschann, Flores, Penilla \& Pantoja, 2006). These studies suggest that differences in the process of enculturation are likely to have a negative impact for families, particularly on parent-adolescent communication and relationships.

Boutakidis, Chao and Rodriguez (2011) looked at the differences in fluency in English and native language in Chinese and Korean families in the United States. The adolescents in these families were either first or second generation Chinese or Korean Americans. Adolescents who were born in the United States (second generation) were found to have greater fluency in English and less fluency in their parents' native language than did first-generation adolescents. Further, it was found that parents had greater fluency in their native language and less fluency in English than their first or second-generation adolescents. Therefore, regardless of generation there was a language disparity between parents and adolescents. Adolescents' fluency in their native language was associated with respect for parents regardless of parents' English proficiency and the quality of their relationships. These researchers explain the results in terms of language being a means for greater shared understanding of cultural heritage between adolescents and their parents.

Shared cultural understanding is important to relationships. For example, Chinese parents tend to show their love through practical support of their children's needs and rely on a mutual understanding of this situation rather than verbal 
communication. On the other hand, western parents tend to express their love through open communication and physical and emotional expression ( $\mathrm{Wu} \& \mathrm{Chao}$, 2011). Therefore, Chinese American adolescents may misinterpret their parents' behaviours as not demonstrating love due to their awareness of western norms of parental behaviour (Wu \& Chao, 2005). Parents who are thoughtful and responsive to their children provide support that decreases the likelihood of adjustment problems regardless of any differences in cultural understanding for Chinese parents and their adolescents ( $\mathrm{Wu} \& \mathrm{Chao}, 2011)$. This finding indicates that responsiveness to adolescents is important for families and adolescent adjustment across cultures.

\subsection{Summary}

Communication is important for parent-adolescent relationships and the development of adolescents. It is a means by which adolescents learn to establish their own opinions and values and assert their individuality. Through communication with their parents, adolescents also learn how to make decisions for themselves.

Parent-adolescent communication can also be an important protective factor for young people. It is helpful for adolescents to discuss what is happening for them with their parents in order to gain assistance and advice; however, adolescents do not readily disclose some areas of their lives to their parents, particularly if they think the issue is personal. Keeping secrets from parents contributes to adolescents' sense of independence, although keeping secrets from parents is also associated with decreased well-being. Adolescents begin to question parents' authority and expect to have more input in decision-making and discipline. They also question the types of issues that should incur parental discipline.

Conflict communication can be both damaging and helpful for adolescents. Poor conflict processes can harm the parent-adolescent relationship, impact negatively on adolescents' behaviour and inhibit their development of problem-solving skills. On the other hand, positive approaches to conflict can be beneficial for parent-adolescent relationships and teach young people how to deal with conflict and assist them to advance their psychosocial development.

Siblings can have a lot of conflict in their relationships and this conflict can be made worse if parents take sides. Adolescents' relationships with their siblings can also be of benefit to them as they can be a valuable support to each other. Peer relationships become important during adolescence and can provide young people with a forum in which they can develop their social competence. Parental supervision and direct involvement with their children's friendships promotes positive peer relationships and the continued development of social competence.

Technology has had a large impact on families and adolescents over recent decades and access to such media can be both beneficial and problematic for them. Most young people have personal mobile phones now, which can be a useful tool 
for keeping in contact with family and friends but can also be a problem when sleep patterns are disturbed because of texting or receiving calls late at night. The internet can be helpful to adolescents for learning thinking skills, for school performance and for communication with family and friends.

\subsection{Implications for Practitioners}

For a number of years, interventions aimed at conflict resolution have been considered an important feature of therapeutic aims for troubled parent-adolescent relationships (e.g. Foster \& Robin, 1998; Greco \& Eifert, 2004). The continued research in the area of parent-adolescent conflict indicates that such a focus remains important; however, the literature also suggests that it is necessary to consider multiple aspects of parentadolescent communication not just conflict processes.

According to the research, the ability of adolescents to discuss ideas and opinions with a responsive and accepting parent is critical for parent-adolescent relationships and for adolescents' ability to develop their own values and opinions. Supportive intimacy in discussions with parents should also facilitate adolescents' learning about problem-solving and negotiating relationships in the broader social world. Therapeutic interventions that include coaching adolescents and parents to discuss an array of topics in a collaborative and accepting manner may enhance both the relationship and adolescent development.

Programs such as Triple P for teens, developed at the University of Queensland by Prof. Matthew Sanders can help both parents and adolescents develop better communication and improve their relationships with one another. Surveys carried out by Prof. Alan Ralph from the same project showed that more than 50 per cent of parents rated talking back to parents and quarrelling with their siblings as their main concerns about their adolescents' behaviour in the family. Almost half (44 per cent) rated adolescents' moodiness and irritability as a serious problem in their families. In addition, a smaller number of parents complained about their adolescents engaging in angry arguments, using abusive or offensive language, and being rebellious and deliberately disobedient. The Teen Triple P (Positive Parenting Program) has been heavily researched and "offers parents ways to avoid or reduce some of the negative aspects of parenting described above and to make parenting teenagers a more positive experience”(see Triple P website).

Another useful program for parents to use with the help of practitioners is the program "Parenting Teenagers" that is part of the Systematic Training for Effective Parenting (STEP; Dinkmeyer, McKay, McKay \& Dinkmeyer, 1998). This program focuses on parents changing their responses to their teenagers, communicating respect and encouragement to them, and using consequences, both natural and logical, to build a sense of responsibility in teenagers. These authors claim in the introduction to their book that: "the rewards of parenting teenagers can far outweigh the challenges. 
With skills and understanding you [a parent] can guide your teen to be responsible, cooperative, and independent. In doing this, you build a strong positive relationship with your teenager. Even parents facing serious problems with teens can find more effective ways to get along with and help their teenager."

Another implication from the research is that for some adolescents and parents with troubled relationships, therapeutic support may be needed in the negotiation of which aspects of adolescents' lives should be primarily under the control of the adolescent as opposed to the control of parents. It is also clear that technology such as mobile phones and the internet can be either enhancing or detrimental for adolescent development and their relationships depending upon how such technology is utilised. Increasing awareness and knowledge about the risks and benefits associated with modern technology is important for adolescents and many parents may not know how best to deal with this issue. Therefore, readily available information and support about the use of technology and the implications for adolescents and their relationships should be made available to families and practitioners.

In this chapter, we explored the important ways in which family members communicate with each other to make a positive contribution to their adolescents' development and adjustment. There is no doubt that communication is very important to family relationships, and parents and adolescents need to listen to each other and be prepared to negotiate with each other for the benefit of the parents, adolescents and for the family as a whole, whatever form that family takes. In the next chapter we discuss adolescents in divorced and reconstituted families. 